\title{
Existence and Ulam stability for nonlinear implicit differential equations with Riemann-Liouville fractional derivative
}

https://doi.org/10.1515/dema-2019-0032

Received April 6, 2019; accepted August 6, 2019

Abstract: In this paper, we establish the existence and uniqueness of solutions for a class of initial value problem for nonlinear implicit fractional differential equations with Riemann-Liouville fractional derivative, also, the stability of this class of problem. The arguments are based upon the Banach contraction principle and Schaefer's fixed point theorem. An example is included to show the applicability of our results.

Keywords: Riemann-Liouville fractional derivative, initial value problem, existence, uniqueness, Ulam stability, fixed point theorem

MSC: $26 \mathrm{~A} 33$

\section{Introduction}

Fractional differential equations have recently proved to be useful tools in the modeling of many physical phenomena. It draws a great application in nonlinear oscillations of earthquakes, many physical phenomena such as seepage ow in porous media and in fluid dynamic traffic model. for more details on fractional calculus theory, one can see [1-7].

Recently, by means of different tools such as the Banach contract principle, Schauder's fixed point, Schaefer's fixed point, the Leray-Schauder nonlinear alternative, Monch fixed point and the measure of noncompactness, initial and boundary value problems for implicit fractional differential equations involving Caputo type fractional derivatives have extensively been studied in the books [8-12] and the papers [13-22].

In $[6,7]$ the existence and uniqueness of solutions of the initial value problem

$$
\begin{aligned}
D_{0^{+}}^{\alpha} u(t)= & f(t, u(t)), \text { for each, } t>0, \\
& D_{0^{+}}^{\alpha-1} u\left(0^{+}\right)=u_{0},
\end{aligned}
$$

was obtained under the assumption that $f:(0, T] \times \mathbb{R} \rightarrow \mathbb{R}$ is Lipchitz continuous, by using the Banach contraction mapping principle. In [23] by using the lower and upper solution method, the authors proved the

\footnotetext{
Mouffak Benchohra: Laboratory of Mathematics, Djillali Liabes University of Sidi Bel-Abbes, P.O. Box 89 Sidi Bel Abbes 22000, Algeria; Department of Mathematics, College of Science, King Saud University, P.O. Box 2455, Riyadh 11451, Saudi Arabia; E-mail: benchohra@yahoo.com

Soufyane Bouriah: Department of Mathematics Faculty of Exact Sciences and Informatics Hassiba Benbouali University, P.O. Box 151 Chlef 02000, Algeria; E-mail: s.bouriah@univ-chlef.dz, bouriahsoufiane@yahoo.fr

*Corresponding Author: Juan J. Nieto: Departamento de Estatistica, Análise Matemática e Optimización, Instituto de Matemáticas, Universidade de Santiago de Compostela, Santiago de Compostela, Spain; E-mail: juanjose.nieto.roig@usc.es
} 
existence of iterative solutions for a class of fractional initial value problem with non-monotone term

$$
\begin{gathered}
D_{0^{+}}^{\alpha} u(t)=f(t, u(t)) \text {, for each, } t \in(0, h), 0<h<+\infty, \\
\left.t^{1-\alpha} u(t)\right|_{t=0}=u_{0} \neq 0 .
\end{gathered}
$$

Motivated by the work above, we focus our attention on the more general problem:

$$
\begin{gathered}
D_{0^{+}}^{\alpha} y(t)=f\left(t, y(t), D_{0^{+}}^{\alpha} y(t)\right), \text { for each, } t \in(0, T], \\
\qquad\left.t^{1-\alpha} y(t)\right|_{t=0}=y_{0} \in \mathbb{R},
\end{gathered}
$$

where $D_{0^{+}}^{\alpha}$ is the standard Riemann-Liouville fractional derivative, $f:(0, T] \times \mathbb{R} \times \mathbb{R} \rightarrow \mathbb{R}$ is a continuous function and $0<\alpha<1$.

In the literature, several different definitions of fractional integrals and derivatives are present. Some of them such as the Riemann-Liouville integral, the Caputo and the Riemann-Liouville derivatives are thoroughly studied and actually used in applied models. Hilfer has introduced a generalized form of the RiemannLiouville fractional derivative. In short, Hilfer fractional derivative $D_{0^{+}}^{\alpha, \beta}$ is an interpolation between the Riemann-Liouville and Caputo fractional derivatives.

The present paper is organized as follows. In Section 2, some notations are introduced and we recall some concepts of preliminaries about fractional calculus and auxiliary results. In Section 3, two results for the problem (1)-(2) are presented: the first one is based on the Banach contraction principle, the second one on Schaefer's fixed point theorem. In Section 4, we present Ulam-Hyers stability result for the problem (1)-(2). Finally, in the last Section, we give an example to illustrate the applicability of our main results.

\section{Preliminaries}

In this section, we introduce notations, definitions, and preliminary facts which are used throughout this paper. Let $0<T, J=[0, T]$. By $C(J, \mathbb{R})$ we denote the Banach space of all continuous functions from $J$ into $\mathbb{R}$ with the norm

$$
\|y\|_{\infty}=\sup \{|y(t)|: t \in J\} .
$$

And $L^{1}(J, \mathbb{R})$ is the space of Lebesgue-integrable functions $w: J \rightarrow \mathbb{R}$ with the norm

$$
\|w\|_{1}=\int_{0}^{T}|w(s)| d s .
$$

$A C^{n}(J)=\left\{h: J \rightarrow \mathbb{R}: h, h^{\prime}, \ldots, h^{(n-1)} \in C(J, \mathbb{R})\right.$ and $h^{(n-1)}$ is absolutely continuous. $\}$

In what follows $\gamma>0$.

We consider the weighted space of continuous functions

$$
C_{\gamma}(J)=\left\{y:(0, T] \rightarrow \mathbb{R}: t^{\gamma} y(t) \in C(J, \mathbb{R})\right\},
$$

with the norm

$$
\|y\|_{C_{\gamma}}=\sup _{t \in J}\left|t^{\gamma} y(t)\right|
$$

Clearly $C_{\gamma}(J)$ is a Banach space. 
Definition 2.1. [6, 24] The fractional (arbitrary) order integral of the function $h \in L^{1}\left([0, T], \mathbb{R}_{+}\right)$of order $\alpha \in \mathbb{R}_{+}$ is defined by

$$
I_{0^{+}}^{\alpha} h(t)=\frac{1}{\Gamma(\alpha)} \int_{0}^{t}(t-s)^{\alpha-1} h(s) d s
$$

where $\Gamma(\cdot)$ is the Euler gamma function defined by $\Gamma(\alpha)=\int_{0}^{\infty} t^{\alpha-1} e^{-t} d t, \alpha>0$.

Lemma 2.2. [25] For $t>0$, we have

$$
\begin{gathered}
{\left[I_{0^{+}}^{\alpha} t^{\beta-1}\right](t)=\frac{\Gamma(\beta)}{\Gamma(\alpha+\beta)} t^{\beta+\alpha-1}, \quad \alpha \geq 0, \beta>0,} \\
{\left[D_{0^{+}}^{\alpha} t^{\alpha-1}\right](t)=0, \quad 0<\alpha<1 .}
\end{gathered}
$$

Definition 2.3. $[6,24]$ For a function $h \in A C^{n}(J)$, the Riemann-Liouville fractional order derivative of order $\alpha$ of $h$, is defined by

$$
D_{0^{+}}^{\alpha} h(t)=\frac{1}{\Gamma(n-\alpha)}\left(\frac{d}{d t}\right)^{n} \int_{0}^{t}(t-s)^{n-\alpha-1} h(s) d s,
$$

where $n=[\alpha]+1$ and $[\alpha]$ denotes the integer part of the real number $\alpha$.

We state the following generalization of Gronwall's Lemma for singular kernels.

Lemma 2.4. [26] Let $\beta>0, \tilde{a}(t)$ be nonnegative function locally integrable on $[a, b)$ and $\tilde{g}(t)$ is a nonnegative, nondecreasing continuous function defined on $\tilde{g}(t)<M, t \in[a, b)$, and suppose $y(t)$ is nonnegative and locally integrable on $[a, b)$ with

$$
y(t) \leq \tilde{a}(t)+\tilde{g}(t) \int_{0}^{t}(t-s)^{\beta-1} y(s) d s, \quad t \in[a, b) .
$$

Then

$$
y(t) \leq \tilde{a}(t)+\int_{0}^{t}\left[\sum_{n=1}^{\infty} \frac{(\tilde{g}(t) \Gamma(\beta))^{n}}{\Gamma(n \beta)}(t-s)^{n \beta-1} \tilde{a}(s)\right] d s, \text { for every } t \in[a, b) .
$$

Corollary 2.5. [26] Under the hypotheses of Lemma 2.4, assume further that $\tilde{a}(t)$ is a nondecreasing function for $t \in[a, b)$. Then

$$
y(t) \leq \tilde{a}(t) E_{\beta}\left(\tilde{g}(t) \Gamma(\beta) t^{\beta}\right), \quad t \in[a, b),
$$

where $E_{\beta}(\cdot)$ is the one parameter Mittag-Leffler function.

For the implicit fractional-order differential equation (1), we adopt the definition in Rus [27] for Ulam-Hyers stability, generalized Ulam-Hyers stability, Ulam-Hyers-Rassias stability and generalized Ulam-Hyers-Rassias stability.

Definition 2.6. The equation (1) is Ulam-Hyers stable if there exists a real number $c_{f}>0$ such that for each $\epsilon>0$ and for each solution $z \in C_{1-\alpha}(J)$ of the inequality

$$
\left|D_{0^{+}}^{\alpha} z(t)-f\left(t, z(t), D_{0^{+}}^{\alpha} z(t)\right)\right| \leq \epsilon, t \in J,
$$

there exists a solution $y \in C_{1-\alpha}(J)$ of equation (1) with

$$
|z(t)-y(t)| \leq c_{f} \epsilon, t \in J .
$$


Definition 2.7. The equation (1) is generalized Ulam-Hyers stable if there exists $\psi_{f} \in C\left(\mathbb{R}_{+}, \mathbb{R}_{+}\right), \psi_{f}(0)=0$, such that for each solution $z \in C_{1-\alpha}(J)$ of the inequality

$$
\left|D_{0^{+}}^{\alpha} z(t)-f\left(t, z(t), D_{0^{+}}^{\alpha} z(t)\right)\right| \leq \epsilon, t \in J,
$$

there exists a solution $y \in C_{1-\alpha}(J)$ of the equation (1) with

$$
|z(t)-y(t)| \leq \psi_{f}(\epsilon), t \in J
$$

Definition 2.8. The equation (1) is Ulam-Hyers-Rassias stable with respect to $\phi \in C\left(J, \mathbb{R}_{+}\right)$if there exists a real number $c_{f}>0$ such that for each $\epsilon>0$ and for each solution $z \in C_{1-\alpha}(J)$ of the inequality

$$
\left|D_{0^{+}}^{\alpha} z(t)-f\left(t, z(t), D_{0^{+}}^{\alpha} z(t)\right)\right| \leq \epsilon \phi(t), t \in J,
$$

there exists a solution $y \in C_{1-\alpha}(J)$ of equation (1) with

$$
|z(t)-y(t)| \leq c_{f} \epsilon \phi(t), t \in J
$$

Definition 2.9. The equation (1) is generalized Ulam-Hyers-Rassias stable with respect to $\phi \in C\left(J, \mathbb{R}_{+}\right)$if there exists a real number $c_{f, \phi}>0$ such that for each solution $z \in C_{1-\alpha}(J)$ of the inequality

$$
\left|D_{0^{+}}^{\alpha} z(t)-f\left(t, z(t), D_{0^{+}}^{\alpha} z(t)\right)\right| \leq \phi(t), t \in J,
$$

there exists a solution $y \in C_{1-\alpha}(J)$ of equation (1) with

$$
|z(t)-y(t)| \leq c_{f, \phi} \phi(t), t \in J .
$$

Remark 2.10. It is clear that:

i) Definition $2.6 \Rightarrow$ Definition 2.7

ii) Definition $2.8 \Rightarrow$ Definition 2.9

iii) Definition 2.8 for $(\phi(t)=\psi=1) \Rightarrow$ Definition 2.6.

Remark 2.11. A function $z \in C_{1-\alpha}(J)$ is a solution of the inequality (4) if and only if there is $\sigma \in C_{1-\alpha}(J)$ (which depends on $z$ ) such that

i) $|\sigma(t)| \leq \epsilon \phi(t), t \in J$

ii) $D_{0^{+}}^{\alpha} z(t)=f\left(t, z(t), D_{0^{+}}^{\alpha} z(t)\right)+\sigma(t), t \in J$.

One can have similar remarks for inequalities (5) and (3).

Theorem 2.12. [28](Theorem of Ascoli-Arzelà) Let $A \subset C_{1-\alpha}(J, \mathbb{R})$. A is relatively compact (i.e $\bar{A}$ is compact) if:

1. A is uniformly bounded i.e, there exists $M>0$ such that

$$
|f(x)|<M \text { for every } f \in A \text { and } x \in J
$$

2. A is equicontinuous i.e, for every $\epsilon>0$, there exists $\delta>0$ such that for each $x, \bar{x} \in J,|x-\bar{x}| \leq \delta$ implies $|f(x)-f(\bar{x})| \leq \epsilon$, for every $f \in A$.

Theorem 2.13. [29] (Banach's fixed point theorem) Let C be a non-empty closed subset of a Banach space E, then any contraction mapping $T$ of $C$ into itself has a unique fixed point.

Theorem 2.14. [29] (Schaefer's fixed point theorem) Let E be a Banach space, and $N: E \longrightarrow E$ completely continuous operator. If the set $\mathcal{E}=\{y \in E: y=\lambda N y$, for some $\lambda \in(0,1)\}$ is bounded, then $N$ has at least one fixed point. 


\section{Existence of solutions}

To prove the existence of solutions to (1)-(2), we need the following auxiliary Lemma.

Lemma 3.1. [6] The linear initial value problem

$$
\left\{\begin{array}{l}
D_{0^{+}}^{\alpha} y(t)+\lambda y(t)=q(t), \text { for each, } t>0 \\
\left.t^{1-\alpha} y(t)\right|_{t=0}=y_{0}
\end{array}\right.
$$

where $\lambda \geq 0$ is a constant and $q \in L(0, T)$, has the following integral representation for a solution

$$
y(t)=\Gamma(\alpha) y_{0} t^{\alpha-1} E_{\alpha, \alpha}\left(-\lambda t^{\alpha}\right)+\int_{0}^{t}(t-s)^{\alpha-1} E_{\alpha, \alpha}\left(-\lambda(t-s)^{\alpha}\right) q(s) d s,
$$

where $E_{\alpha, \alpha}(t)$ is the two-parameter Mittag-Leffler function. In particular, when $\lambda=0$, then the initial value problem (6) has a unique solution defined by

$$
y(t)=y_{0} t^{\alpha-1}+\frac{1}{\Gamma(\alpha)} \int_{0}^{t}(t-s)^{\alpha-1} q(s) d s .
$$

Lemma 3.2. [23] For $0<\alpha \leq 1$; the Mittag-Leffler type function $E_{\alpha, \alpha}\left(-\lambda t^{\alpha}\right)$ satisfies

$$
0 \leq E_{\alpha, \alpha}\left(-\lambda t^{\alpha}\right) \leq \frac{1}{\Gamma(\alpha)}, t \in[0, \infty), \lambda \geq 0,
$$

and

$$
\lim _{t \rightarrow 0^{+}} E_{\alpha, \alpha}\left(-\lambda t^{\alpha}\right)=E_{\alpha, \alpha}(0)=\frac{1}{\Gamma(\alpha)} .
$$

The following assumptions will be used in our main results:

(H1) There exist constants $K>0$ and $0<L<1$ such that

$$
|f(t, u, v)-f(t, \bar{u}, \bar{v})| \leq K|u-\bar{u}|+L|v-\bar{v}|
$$

for any $u, v, \bar{u}, \bar{v} \in \mathbb{R}$ and $t \in(0, T]$.

Theorem 3.3. Suppose (H1) holds. The function y solves problem (1)-(2) if and only if it is a fixed-point of the operator $N: C_{1-\alpha}(J) \rightarrow C_{1-\alpha}(J)$ defined by

$$
N y(t)=y_{0} t^{\alpha-1}+\frac{1}{\Gamma(\alpha)} \int_{0}^{t}(t-s)^{\alpha-1} g(s) d s, t \in(0, T],
$$

where $g:(0, T] \rightarrow \mathbb{R}$ be a function satisfying the functional equation

$$
g(t)=f(t, y(t), g(t)) .
$$

Proof. Firstly, we need to show that the operator $N$ is well defined, i.e, for every $y \in C_{1-\alpha}(J)$ and $t>0$; the integral

$$
\frac{1}{\Gamma(\alpha)} \int_{0}^{t}(t-s)^{\alpha-1} g(s) d s,
$$

belongs to $C_{1-\alpha}(J)$. Under condition $\left(\mathrm{H}_{1}\right)$,

$$
|g(t)| \leq \frac{K}{1-L}|y(t)|+C, \text { for each, } t \in J,
$$


where

$$
C=\frac{\sup _{t \in J}|f(t, 0,0)|}{1-L} .
$$

By Lemma 3.2, for $y \in C_{1-\alpha}(J)$, we have

$$
\begin{aligned}
\left|\frac{t^{1-\alpha}}{\Gamma(\alpha)} \int_{0}^{t}(t-s)^{\alpha-1} g(s) d s\right| & \leq \frac{t^{1-\alpha}}{\Gamma(\alpha)} \int_{0}^{t}(t-s)^{\alpha-1}|g(s)| d s \\
& \leq \frac{t^{1-\alpha}}{\Gamma(\alpha)} \int_{0}^{t}(t-s)^{\alpha-1}\left[\frac{K}{1-L}|y(s)|+C\right] d s \\
& \leq \frac{t^{1-\alpha}}{\Gamma(\alpha)} \int_{0}^{t}(t-s)^{\alpha-1} s^{\alpha-1} \frac{K}{1-L}\left|s^{1-\alpha} y(s)\right| d s+\frac{t^{1-\alpha} T^{\alpha} C}{\alpha \Gamma(\alpha)} \\
& \leq t^{1-\alpha}\|y\|_{C_{1-\alpha}} \frac{K}{1-L} I_{0^{+}}^{\alpha}\left(t^{\alpha-1}\right)+\frac{t^{1-\alpha} T^{\alpha} C}{\Gamma(\alpha+1)} .
\end{aligned}
$$

By Lemma 2.2, we have

$$
\begin{aligned}
\left|\frac{t^{1-\alpha}}{\Gamma(\alpha)} \int_{0}^{t}(t-s)^{\alpha-1} g(s) d s\right| & \leq \frac{K \Gamma(\alpha) t^{\alpha}\|y\|_{C_{1-\alpha}}}{\Gamma(2 \alpha)(1-L)}+\frac{T C}{\Gamma(\alpha+1)} \\
& \leq \frac{K \Gamma(\alpha) T^{\alpha}\|y\|_{C_{1-\alpha}}}{\Gamma(2 \alpha)(1-L)}+\frac{T C}{\Gamma(\alpha+1)} .
\end{aligned}
$$

That is to say that the integral exists and belongs to $C_{1-\alpha}(J)$.

The above inequality implies that

$$
\lim _{t \rightarrow 0^{+}} \frac{t^{1-\alpha}}{\Gamma(\alpha)} \int_{0}^{t}(t-s)^{\alpha-1} g(s) d s=0
$$

Combining with $\lim _{t \rightarrow 0^{+}} t^{1-\alpha}(N y)(t)=y_{0}$. The above arguments combined with Lemma 3.1, imply that the fixedpoint of the operator $N$ solves (1)-(2) and vice versa. The proof is complete.

Theorem 3.4. Suppose (H1) holds. If

$$
\frac{K \Gamma(\alpha) T^{\alpha}}{\Gamma(2 \alpha)(1-L)}<1
$$

then, there exists a unique solution for the problem (1)-(2) in the space $C_{1-\alpha}(J)$.

Proof. Let $u, w \in C_{1-\alpha}(J)$. Then for $t \in(0, T]$, we have

$$
|N u(t)-N w(t)| \leq \frac{1}{\Gamma(\alpha)} \int_{0}^{t}(t-s)^{\alpha-1}|g(s)-h(s)| d s,
$$

where $g, h \in C_{1-\alpha}(J)$ be such that

$$
g(t)=f(t, u(t), g(t))
$$

and

$$
h(t)=f(t, w(t), h(t)) .
$$

By (H1) we have

$$
|g(t)-h(t)|=|f(t, u(t), g(t))-f(t, w(t), h(t))|
$$




$$
\leq \quad K|u(t)-w(t)|+L|g(t)-h(t)| \text {. }
$$

Then

$$
|g(t)-h(t)| \leq \frac{K}{1-L}|u(t)-w(t)| .
$$

Therefore, for each $t \in(0, T]$

$$
\begin{aligned}
|N u(t)-N w(t)| & \leq \frac{K}{\Gamma(\alpha)(1-L)} \int_{0}^{t}(t-s)^{\alpha-1}|u(s)-w(s)| d s \\
& =\frac{K}{\Gamma(\alpha)(1-L)} \int_{0}^{t}(t-s)^{\alpha-1} s^{\alpha-1}\left|s^{1-\alpha}(u(s)-w(s))\right| d s \\
& \leq \frac{K}{1-L} I_{0^{+}}^{\alpha}\left(t^{\alpha-1}\right)\|u-w\|_{C_{1-\alpha}} .
\end{aligned}
$$

By Lemma 2.2, we have

$$
|N u(t)-N w(t)| \leq \frac{\Gamma(\alpha) K t^{2 \alpha-1}}{\Gamma(2 \alpha)(1-L)}\|u-w\|_{C_{1-\alpha}}
$$

which implies that

$$
\begin{aligned}
\left|t^{1-\alpha}(N u(t)-N w(t))\right| & \leq \frac{\Gamma(\alpha) K t^{\alpha}}{\Gamma(2 \alpha)(1-L)}\|u-w\|_{C_{1-\alpha}} \\
& \leq \frac{\Gamma(\alpha) K T^{\alpha}}{\Gamma(2 \alpha)(1-L)}\|u-w\|_{C_{1-\alpha}}
\end{aligned}
$$

Thus

$$
\|N u-N w\|_{C_{1-\alpha}} \leq \frac{\Gamma(\alpha) K T^{\alpha}}{\Gamma(2 \alpha)(1-L)}\|u-w\|_{C_{1-\alpha}} .
$$

By (8), the operator $T$ is a contraction. Hence, by Banach's contraction principle, $N$ has a unique fixed point which is a unique solution of the problem (1)-(2).

Our second result is based on Schaefer's fixed point theorem.

Theorem 3.5. Assume (H1) and

(H2) There exist $p, q, r \in C\left(J, \mathbb{R}_{+}\right)$with $r^{\star}=\sup _{t \in J} r(t)<1$ such that

$$
|f(t, u, w)| \leq p(t)+q(t)|u|+r(t)|w| \text { for } t \in J \text { and } u, w \in \mathbb{R}
$$

If

$$
\frac{q^{\star} \Gamma(\alpha) T^{\alpha}}{\Gamma(2 \alpha)\left(1-r^{\star}\right)}<1
$$

where $q^{\star}=\sup _{t \in J} q(t)$ then the problem (1)-(2) has at least one solution in the space $C_{1-\alpha}(J)$.

Proof. Let the operator $N$ defined in (7). We shall use Schaefer's fixed point theorem to prove that $N$ has a fixed point. The proof will be given in several steps.

Step 1: $N$ is continuous. Let $\left(y_{n}\right)_{n \in \mathbb{N}}$ be a sequence such that $y_{n} \rightarrow y$ in $C_{1-\alpha}(J)$, then for each $t \in(0, T]$, we have

$$
\left|N y_{n}(t)-N y(t)\right| \leq \frac{1}{\Gamma(\alpha)} \int_{0}^{t}(t-s)^{\alpha-1}\left|g_{n}(s)-g(s)\right| d s
$$

where $g_{n}, g \in C_{1-\alpha}(J)$ be such that

$$
g_{n}(t)=f\left(t, y_{n}(t), g_{n}(t)\right)
$$


and

$$
g(t)=f(t, y(t), g(t))
$$

By (H1) we have

$$
\begin{aligned}
\left|g_{n}(t)-g(t)\right| & =\left|f\left(t, y_{n}(t), g_{n}(t)\right)-f(t, y(t), g(t))\right| \\
& \leq K\left|y_{n}(t)-y(t)\right|+L\left|g_{n}(t)-g(t)\right| .
\end{aligned}
$$

Then

$$
\left|g_{n}(t)-g(t)\right| \leq \frac{K}{1-L}\left|y_{n}(t)-y(t)\right| .
$$

By replacing (11) in inequality (10), we find

$$
\begin{aligned}
\left|N y_{n}(t)-N y(t)\right| & \leq \frac{K}{\Gamma(\alpha)(1-L)} \int_{0}^{t}(t-s)^{\alpha-1}\left|y_{n}(s)-y(s)\right| d s \\
& =\frac{K}{\Gamma(\alpha)(1-L)} \int_{0}^{t}(t-s)^{\alpha-1} s^{\alpha-1}\left|s^{1-\alpha}\left(y_{n}(s)-y(s)\right)\right| d s \\
& \leq \frac{K}{1-L} I_{0^{+}}^{\alpha}\left(t^{\alpha-1}\right)\left\|y_{n}-y\right\|_{C_{1-\alpha}}
\end{aligned}
$$

By Lemma 2.2, we have

$$
\left|N y_{n}(t)-N y(t)\right| \leq \frac{\Gamma(\alpha) K t^{2 \alpha-1}}{\Gamma(2 \alpha)(1-L)}\left\|y_{n}-y\right\|_{C_{1-\alpha}},
$$

which implies that

$$
\begin{aligned}
\left|t^{1-\alpha}\left(N y_{n}(t)-N y(t)\right)\right| & \leq \frac{\Gamma(\alpha) K t^{\alpha}}{\Gamma(2 \alpha)(1-L)}\left\|y_{n}-y\right\|_{C_{1-\alpha}} \\
& \leq \frac{\Gamma(\alpha) K T^{\alpha}}{\Gamma(2 \alpha)(1-L)}\left\|y_{n}-y\right\|_{C_{1-\alpha}} .
\end{aligned}
$$

Thus

$$
\left|t^{1-\alpha}\left(N y_{n}(t)-N y(t)\right)\right| \rightarrow 0 \text { as } n \rightarrow \infty,
$$

and hence

$$
\left\|N y_{n}-N y\right\|_{C_{1-\alpha}} \rightarrow 0 \text { as } n \rightarrow \infty .
$$

Consequently, $N$ is continuous.

Step 2: $N$ maps bounded sets into bounded sets in $C_{1-\alpha}$. Indeed, it is enough to show that for any $\eta^{\star}>0$, there exists a positive constant $\ell$ such that for each

$$
y \in B_{\eta^{*}}=\left\{y \in C_{1-\alpha}(J):\|y\|_{C_{1-\alpha}} \leq \eta^{*}\right\},
$$

we have $\|N y\|_{C_{1-\alpha}} \leq \ell$. For each $t \in(0, T]$, we have

$$
N y(t)=y_{0} t^{\alpha-1}+\frac{1}{\Gamma(\alpha)} \int_{0}^{t}(t-s)^{\alpha-1} g(s) d s,
$$

where $g \in C_{1-\alpha}(J)$ be such that

$$
g(t)=f(t, y(t), g(t))
$$

By (H2), we have for each $t \in(0, T]$,

$$
|g(t)|=|f(t, y(t), g(t))| \leq p(t)+q(t)|y(t)|+r(t)|g(t)| .
$$


Hence, we get

$$
\begin{aligned}
\left|t^{1-\alpha} g(t)\right| & \leq t^{1-\alpha} p(t)+q(t)\left|t^{1-\alpha} y(t)\right|+r(t)\left|t^{1-\alpha} g(t)\right| \\
& \leq T^{1-\alpha} p^{\star}+q^{\star} \eta^{\star}+r^{\star}\left|t^{1-\alpha} g(t)\right|
\end{aligned}
$$

where $p^{\star}=\sup \{p(t), t \in J\}$, Then, for each $t \in(0, T]$, we have

$$
\left|t^{1-\alpha} g(t)\right| \leq \frac{T^{1-\alpha} p^{\star}+q^{\star} \eta^{\star}}{1-r^{\star}}:=M .
$$

Thus, (12) implies

$$
\begin{aligned}
|N y(t)| & \leq t^{\alpha-1}\left|y_{0}\right|+\frac{M}{\Gamma(\alpha)} \int_{0}^{t}(t-s)^{\alpha-1} s^{\alpha-1} d s \\
& \leq t^{\alpha-1}\left|y_{0}\right|+\frac{M \Gamma(\alpha)}{\Gamma(2 \alpha)} t^{2 \alpha-1} .
\end{aligned}
$$

Therefore

$$
\left|t^{1-\alpha} N y(t)\right| \leq\left|y_{0}\right|+\frac{M \Gamma(\alpha)}{\Gamma(2 \alpha)} T^{\alpha}
$$

Thus

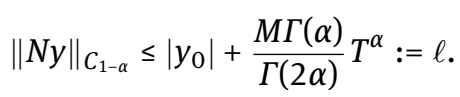

Step 3: $N$ maps bounded sets into equicontinuous sets of $C_{1-\alpha}(J)$. Let $0<t_{1}<t_{2} \leq T, B_{\eta^{\star}}$ be a bounded set of $C_{1-\alpha}(J)$ as in Step 2, and let $y \in B_{\eta^{\star}}$. Then

$$
\begin{aligned}
\left|t_{2}^{1-\alpha} N y\left(t_{2}\right)-t_{1}^{1-\alpha} N y\left(t_{1}\right)\right|= & \frac{1}{\Gamma(\alpha)}\left|\int_{0}^{t_{2}} t_{2}^{1-\alpha}\left(t_{2}-s\right)^{\alpha-1} g(s) d s-\int_{0}^{t_{1}} t_{1}^{1-\alpha}\left(t_{1}-s\right)^{\alpha-1} g(s) d s\right| \\
= & \frac{1}{\Gamma(\alpha)} \mid \int_{0}^{t_{1}} t_{2}^{1-\alpha}\left(t_{2}-s\right)^{\alpha-1} s^{\alpha-1}\left(s^{1-\alpha} g(s)\right) d s+\int_{t_{1}}^{t_{2}} t_{2}^{1-\alpha}\left(t_{2}-s\right)^{\alpha-1} s^{\alpha-1}\left(s^{1-\alpha} g(s)\right) d s \\
& -\int_{0}^{t_{1}} t_{1}^{1-\alpha}\left(t_{1}-s\right)^{\alpha-1} s^{\alpha-1}\left(s^{1-\alpha} g(s)\right) d s \mid \\
\leq & \frac{1}{\Gamma(\alpha)} \int_{0}^{t_{1}}\left|t_{2}^{1-\alpha}\left(t_{2}-s\right)^{\alpha-1} s^{\alpha-1}-t_{1}^{1-\alpha}\left(t_{1}-s\right)^{\alpha-1} s^{\alpha-1}\right|\left|s^{1-\alpha} g(s)\right| d s \\
& +\frac{1}{\Gamma(\alpha)} \int_{t_{1}}^{t_{2}} t_{2}^{1-\alpha}\left(t_{2}-s\right)^{\alpha-1} s^{\alpha-1}\left|s^{1-\alpha} g(s)\right| d s \\
& \frac{M}{\Gamma(\alpha)} \int_{0}^{t_{1}}\left|t_{2}^{1-\alpha}\left(t_{2}-s\right)^{\alpha-1}-t_{1}^{1-\alpha}\left(t_{1}-s\right)^{\alpha-1}\right| s^{\alpha-1} d s \\
& +\frac{M}{\Gamma(\alpha)} \int_{t_{1}}^{t_{2}} t_{2}^{1-\alpha}\left(t_{2}-s\right)^{\alpha-1} s^{\alpha-1} d s .
\end{aligned}
$$

As $t_{1} \rightarrow t_{2}$, the right-hand side of the above inequality tends to zero. That is to say that $T\left(B_{\eta^{*}}\right)$ is equicontinuous. As a consequence of Step 1 to Step 3 together with the Ascoli-Arzela theorem, we can conclude that $T: C_{1-\alpha}(J) \rightarrow C_{1-\alpha}(J)$ is completely continuous.

Step 4: A priori bounds. Now it remains to show that the set

$$
E=\left\{y \in C_{1-\alpha}: y=\lambda N(y) \text { for some } 0<\lambda<1\right\},
$$


is bounded. Let $y \in E$, then $y=\lambda N y$ for some $0<\lambda<1$. Thus, for each $t \in(0, T]$, we have

$$
y(t)=\lambda y_{0} t^{\alpha-1}+\frac{\lambda}{\Gamma(\alpha)} \int_{0}^{t}(t-s)^{\alpha-1} g(s) d s .
$$

And by (H2), we have for each $t \in(0, T]$,

$$
|g(t)| \leq p(t)+q(t)|y(t)|+r(t)|g(t)| .
$$

Thus, we get

$$
\begin{aligned}
\left|t^{1-\alpha} g(t)\right| & \leq t^{1-\alpha} p(t)+q(t)\left|t^{1-\alpha} y(t)\right|+r(t)\left|t^{1-\alpha} g(t)\right| \\
& \leq T^{1-\alpha} p^{*}+q^{*}\|y\|_{C_{1-\alpha}}+r^{*}\left|t^{1-\alpha} g(t)\right|,
\end{aligned}
$$

then

$$
\left|t^{1-\alpha} g(t)\right| \leq \frac{T^{1-\alpha} p^{\star}}{1-r^{\star}}+\frac{q^{\star}}{1-r^{\star}}\|y\|_{C_{1-\alpha}} .
$$

This implies, by (13) and Lemma 2.2, that for each $t \in(0 ; T]$ we have

$$
\begin{aligned}
|y(t)| & \leq\left|y_{0}\right| t^{\alpha-1}+\frac{T^{1-\alpha} p^{\star}}{\left(1-r^{\star}\right) \Gamma(\alpha)} \int_{0}^{t}(t-s)^{\alpha-1} s^{\alpha-1} d s+\frac{q^{\star}\|y\|_{C_{1-\alpha}}}{\left(1-r^{\star}\right) \Gamma(\alpha)} \int_{0}^{t}(t-s)^{\alpha-1} s^{\alpha-1} d s \\
& \leq\left|y_{0}\right| t^{\alpha-1}+\frac{T^{1-\alpha} p^{\star} \Gamma(\alpha)}{\left(1-r^{\star}\right) \Gamma(2 \alpha)} t^{2 \alpha-1}+\frac{q^{\star} \Gamma(\alpha)}{\left(1-r^{\star}\right) \Gamma(2 \alpha)} t^{2 \alpha-1}\|y\|_{C_{1-\alpha}} .
\end{aligned}
$$

Therefore

$$
\left|t^{1-\alpha} y(t)\right| \leq\left|y_{0}\right|+\frac{T p^{\star} \Gamma(\alpha)}{\left(1-r^{\star}\right) \Gamma(2 \alpha)}+\frac{q^{\star} T^{\alpha} \Gamma(\alpha)}{\left(1-r^{\star}\right) \Gamma(2 \alpha)}\|y\|_{C_{1-\alpha}} .
$$

Finally, by (9) we have

$$
\|y\|_{C_{1-\alpha}} \leq \frac{\left(1-r^{\star}\right) \Gamma(2 \alpha)\left|y_{0}\right|+T p^{\star} \Gamma(\alpha)}{\left(1-r^{\star}\right) \Gamma(2 \alpha)-q^{\star} T^{\alpha} \Gamma(\alpha)} .
$$

This shows that the set $E$ is bounded. As a consequence of Schaefer's fixed point theorem, we deduce that $N$ has at least a fixed point $y^{\star} \in C_{1-\alpha}(J)$ which is a solution of the problem (1)-(2).

\section{Ulam-Hyers-Rassias stability}

Now, we state the following Ulam-Hyers-Rassias stable result.

Theorem 4.1. Assume (H1), (8) and

(H3) there exists a nondecreasing function $\phi \in C\left(J, \mathbb{R}^{+}\right)$and there exists $\lambda_{\phi}>0$ such that for any $t \in(0, T]$ :

$$
{ }^{\rho} I_{0^{+}}^{\alpha} \phi(t) \leq \lambda_{\phi} \phi(t)
$$

are satisfied, then, the equation (1) is Ulam-Hyers-Rassias stable with respect to $\phi$.

Proof. Let $z \in C_{1-\alpha}(J)$ be a solution of the inequality (4). Denote by $y$ the unique solution of the initial value problem:

$$
\begin{gathered}
D_{0^{+}}^{\alpha} y(t)=f\left(t, y(t), D_{0^{+}}^{\alpha} y(t)\right), \text { for each }, t \in(0, T], \\
\left.t^{1-\alpha} y(t)\right|_{t=0}=y_{0} .
\end{gathered}
$$

Using Theorem 3.3, we obtain for each $t \in(0, T]$

$$
y(t)=t^{1-\alpha} y_{0}+\frac{1}{\Gamma(\alpha)} \int_{0}^{t}(t-s)^{\alpha-1} g(s) d s,
$$


where $g:(0, T] \rightarrow \mathbb{R}$ be a function satisfying the functional equation

$$
g(t)=f(t, y(t), g(t)) .
$$

Since $z$ solution of the inequality (4) and by Remark 2.11, we have

$$
\begin{gathered}
D_{0^{+}}^{\alpha} z(t)=f\left(t, z(t), D_{0^{+}}^{\alpha} z(t)\right)+\sigma(t), \text { for each }, t \in(0, T], \\
\qquad\left.t^{1-\alpha} z(t)\right|_{t=0}=y_{0} .
\end{gathered}
$$

Clearly, the solution of the problem (14)-(15) is given by

$$
z(t)=t^{1-\alpha} y_{0}+\frac{1}{\Gamma(\alpha)} \int_{0}^{t}(t-s)^{\alpha-1}(h(s)+\sigma(s)) d s, t \in(0, T],
$$

where $h:(0, T] \rightarrow \mathbb{R}$ be a function satisfying the functional equation

$$
h(t)=f(t, z(t), h(t)+\sigma(t)) .
$$

Hence for each $t \in(0, T]$, it follows that

$$
\begin{aligned}
|z(t)-y(t)| & \leq \frac{1}{\Gamma(\alpha)} \int_{0}^{t}(t-s)^{\alpha-1}|\sigma(s)| d s+\frac{1}{\Gamma(\alpha)} \int_{0}^{t}(t-s)^{\alpha-1}|h(s)-g(s)| d s \\
& \leq \frac{\epsilon}{\Gamma(\alpha)} \int_{0}^{t}(t-s)^{\alpha-1} \phi(s) d s+\frac{1}{\Gamma(\alpha)} \int_{0}^{t}(t-s)^{\alpha-1}|h(s)-g(s)| d s \\
& \leq \epsilon \lambda_{\phi} \phi(t)+\frac{1}{\Gamma(\alpha)} \int_{0}^{t}(t-s)^{\alpha-1}|h(s)-g(s)| d s,
\end{aligned}
$$

where $g, h \in C_{1-\alpha}(J)$ such that

$$
\begin{aligned}
& g(t)=f(t, y(t), g(t)), \\
& h(t)=f(t, z(t), h(t)+\sigma(t)) .
\end{aligned}
$$

By (H1), we have

$$
\begin{aligned}
|h(t)-g(t)| & =|f(t, z(t), h(t)+\sigma(t))-f(t, y(t), g(t))| \\
& \leq K|z(t)-y(t)|+L|h(t)-g(t)|+L|\sigma(t)| .
\end{aligned}
$$

Then,

$$
|h(t)-g(t)| \leq \frac{K}{1-L}|z(t)-y(t)|+\frac{L}{1-L}|\sigma(t)| .
$$

Therefore, for each $t \in(0, T]$

$$
|z(t)-y(t)| \leq \frac{\epsilon \lambda_{\phi} \phi(t)}{1-L}+\frac{K}{(1-L) \Gamma(\alpha)} \int_{0}^{t}(t-s)^{\alpha-1}|z(s)-y(s)| d s .
$$

Applying Corollary 2.5, we get

$$
\begin{aligned}
|z(t)-y(t)| & \leq \frac{\epsilon \lambda_{\phi} \phi(t)}{1-L} E_{\alpha}\left(\frac{K t^{\alpha}}{1-L}\right) \\
& \leq \frac{\epsilon \lambda_{\phi} \phi(t)}{1-L} E_{\alpha}\left(\frac{K T^{\alpha}}{1-L}\right)=\epsilon c_{\phi} \phi(t),
\end{aligned}
$$


where

$$
c_{\phi}=\frac{\lambda_{\phi}}{1-L} E_{\alpha}\left(\frac{K T^{\alpha}}{1-L}\right) .
$$

Thus, the equation (1) is Ulam-Hyers-Rassias stable with respect to $\phi$. The proof is complete.

Next, we present the following Ulam-Hyers stable result.

Theorem 4.2. Assume that (H1) and (8) are satisfied, then, the equation (1) is Ulam-Hyers stable.

Proof. Let $z \in C_{1-\alpha}(J)$ be a solution of the inequality (3). Denote by $y$ the unique solution of the initial value problem:

$$
\begin{gathered}
D_{0^{+}}^{\alpha} y(t)=f\left(t, y(t), D_{0^{+}}^{\alpha} y(t)\right), \text { for each }, t \in(0, T], \\
\left.t^{1-\alpha} y(t)\right|_{t=0}=y_{0} .
\end{gathered}
$$

By the same way of the proof of Theorem 4.1, we can easily show that

$$
\begin{aligned}
|z(t)-y(t)| & \leq \frac{1}{\Gamma(\alpha)} \int_{0}^{t}(t-s)^{\alpha-1}|\sigma(s)| d s+\frac{1}{\Gamma(\alpha)} \int_{0}^{t}(t-s)^{\alpha-1}|h(s)-g(s)| d s \\
& \leq \frac{\epsilon}{\Gamma(\alpha)} \int_{0}^{t}(t-s)^{\alpha-1} d s+\frac{1}{\Gamma(\alpha)} \int_{0}^{t}(t-s)^{\alpha-1}|h(s)-g(s)| d s \\
& \leq \frac{\epsilon T^{\alpha}}{\Gamma(\alpha+1)}+\frac{1}{\Gamma(\alpha)} \int_{0}^{t}(t-s)^{\alpha-1}|h(s)-g(s)| d s \\
& \leq \frac{\epsilon T^{\alpha}}{\Gamma(\alpha+1)(1-L)}+\frac{K}{\Gamma(\alpha)(1-L)} \int_{0}^{t}(t-s)^{\alpha-1}|z(s)-y(s)| d s .
\end{aligned}
$$

Applying Lemma 2.4, we get

$$
\begin{aligned}
|z(t)-y(t)| & \leq \frac{\epsilon T^{\alpha}}{\Gamma(\alpha+1)(1-L)}\left[1+\int_{0}^{t} \sum_{n=1}^{\infty} \frac{\left(\frac{K}{1-L}\right)^{n}}{\Gamma(n \alpha)}(t-s)^{n \alpha-1} d s\right] \\
& \leq \frac{\epsilon T^{\alpha}}{\Gamma(\alpha+1)(1-L)}\left[1+\sum_{n=1}^{\infty} \frac{\left(\frac{K T^{\alpha}}{1-L}\right)^{n}}{\Gamma(n \alpha+1)}\right] \\
& =\frac{\epsilon T^{\alpha}}{\Gamma(\alpha+1)(1-L)} E_{\alpha}\left(\frac{K T^{\alpha}}{1-L}\right)=c \epsilon,
\end{aligned}
$$

which completes the proof of the theorem.

Moreover, if we set $\psi_{f}(\epsilon)=c \epsilon ; \psi_{f}(0)=0$, then, the equation (1) is generalized Ulam-Hyers stable.

\section{An example}

Consider the following initial value problem

$$
D_{0^{+}}^{\frac{1}{2}} y(t)=\frac{1}{10 e^{-t+2}\left(1+|y(t)|+\left|D_{0^{+}}^{\frac{1}{2}} y(t)\right|\right)}+\frac{1}{\sqrt{t}} \text { for each } t \in(0,1]
$$




$$
\left.t^{\frac{1}{2}} y(t)\right|_{t=0}=\frac{3}{2}
$$

Set

$$
f(t, u, v)=\frac{1}{10 e^{-t+2}(1+|u|+|v|)}+\frac{1}{\sqrt{t}}, \quad t \in(0,1], u, v \in \mathbb{R}
$$

We have

$$
C_{1-\alpha}([0,1])=C_{\frac{1}{2}}([0,1])=\left\{h:(0,1] \rightarrow \mathbb{R}: t^{\frac{1}{2}} h \in C([0,1])\right\},
$$

with $\alpha=\frac{1}{2}$. Clearly, the function $f \in C_{\frac{1}{2}}([0,1])$.

For each $u, \bar{u}, v, \bar{v} \in \mathbb{R}$ and $t \in(0,1]$, we have

$$
|f(t, u, v)-f(t, \bar{u}, \bar{v})| \leq \frac{1}{10 e}(|u-\bar{u}|+|v-\bar{v}|) .
$$

Hence, condition (H1) is satisfied with $K=L=\frac{1}{10 e}$.

The condition

$$
\frac{K T^{\alpha} \Gamma(\gamma)}{(1-L) \Gamma(2 \alpha)}=\frac{\sqrt{\pi}}{10 e-1}<1,
$$

is satisfied with $T=1$. It follows from Theorem 3.5 that the problem (16)-(17) has a unique solution in the space $C_{\frac{1}{2}}([0,1])$. Moreover, Theorem 4.2 implies that the equation (16) is Ulam-Hyers stable.

Acknowledgements: The research of J.J. Nieto has been partially supported by the AEI of Spain under Grant MTM2016-75140-P and co-financed by European Community fund FEDER.

\section{References}

[1] Capelas de Oliveira E., Vanterler da C. Sousa J., Leibniz type rule: $\psi$-Hilfer fractional operator, Comm. Nonl. Sci. Numer. Simul., 2019, 77, 305-311

[2] Capelas de Oliveira E., Vanterler da C. Sousa J., Ulam-Hyers stability of a nonlinear fractional Volterra integro-differential equation, Appl. Math. Let., 2018, 81, 50-56

[3] Bai Z., On positive solutions of a nonlocal fractional boundary value problem, Nonlinear Anal., 2010, 72, 916-924

[4] Bai Z., Chen Y., Sun S., Lian H., On the existence of blow up solutions for a class of fractional differential equations, Frac. Calc. Anal., 2014, 17, 1175-1187

[5] Bai Z., Lu H., Positive solutions of boundary value problems of nonlinear fractional differential equation, J. Math. Anal. Appl., 2005, 311, 495-505

[6] Kilbas A. A., Srivastava Hari M., Trujillo Juan J., Theory and Applications of Fractional Differential Equations, North-Holland Mathematics Studies, 204, Elsevier Science B.V., Amsterdam, 2006

[7] Samko S. G., Kilbas A. A., Marichev O.nl., Fractional Integrals and Derivatives, Theory and Applications, Gordon and Breach, Amsterdam, 1993

[8] Abbas S., Benchohra M., Graef J. R., Henderson J., Implicit Fractional Differential and Integral Equations: Existence and Stability, De Gruyter, Berlin, 2018

[9] Abbas S., Benchohra M., N’Guérékata G. M., Topics in Fractional Differential Equations, Springer-Verlag, New York, 2012

[10] Abbas S., Benchohra M., N'Guérékata G. M., Advanced Fractional Differential and Integral Equations, Nova Science Publishers, New York, 2014

[11] Ahmad B., Alsaedi A., Ntouyas S. K., Tariboon J., Hadamard-type Fractional Differential Equations, Inclusions and Inequalities, Springer, Cham, 2017

[12] Zhou Y., Wang J.-R., Zhang L., Basic Theory of Fractional Differential Equations, Second edition, World Scientific Publishing Co. Pte. Ltd., Hackensack, NJ, 2017

[13] Abbas S., Benchohra M., Bouriah S., Nieto J. J., Periodic solutions for nonlinear fractional differential systems, Differ. Equ. Appl., 2018, 10(3), 299-316

[14] Ahmad B., Nieto J. J., Existence results for nonlinear boundary value problems of fractional integrodifferential equations with integral boundary conditions, Boundary Value Problems, 2009, Article ID 708576

[15] Benchohra M., Bouriah S., Existence and stability results for nonlinear boundary value problem for implicit differential equations of fractional order, Moroccan J. Pure. Appl. Anal., 2015, 1, 22-36

[16] Benchohra M., Bouriah S., Existence and stability results for neutral functional differential equations of fractional order with delay, Dyn. Contin., Discrete Impul. Syst. Series A: Math. Anal., 2016, 23, 295-307 
[17] Benchohra M., Bouriah S., Darwish M. A., Nonlinear boundary value problem for implicit differential equations of fractional order in Banach spaces, Fixed Point Theory, 2017, 18, 457-470

[18] Benchohra M., Bouriah S., Graef J. R., Boundary value problems for nonlinear implicit Caputo-Hadamard type fractional differential equations with impulses, Mediterr. J. Math., 2017, 14:206

[19] Benchohra M., Bouriah S., Graef J. R., Nonlinear implicit differential equations of fractional order at resonance, Electron. J. Differential Equations, 2016, 2016(324), 1-10

[20] Benchohra M., Bouriah S., Henderson J., Existence and stability results for nonlinear implicit neutral fractional differential equations with finite delay and impulses, Comm. Appl. Nonlinear Anal., 2015, 22(1), 46-67

[21] Benchohra M., Lazreg J. E., Nonlinear fractional implicit differential equations, Commun. Appl. Anal., 2013, 17, 471-482

[22] Benchohra M., Lazreg J. E., Existence and uniqueness results for nonlinear implicit fractional differential equations with boundary conditions, Rom. J. Math. Comput. Sc., 2014, 4, 60-72

[23] Bai Z., Zhang S., Sun S., Yin C., Monotone iterative method for fractional differential equations, Electron. J. Differential Equations, 2016, 2016(06), 1-8

[24] Podlubny I., Fractional Differential Equations, Academic Press, San Diego, 1999

[25] Furati K. M., Kassim M. D., Tahar N.-E., Existence and uniqueness for a problem involving Hilfer fractional derivative, Comput. Math. Appl., 2012, 64, 1616-1626

[26] Ye H., Gao J., Ding Y., A generalized Gronwall inequality and its application to a fractional differential equation, J. Math. Anal. Appl., 2007, 328, 1075-1081

[27] Rus I. A., Ulam stabilities of ordinary differential equations in a Banach space, Carpathian J. Math., 2010, 26, 103-107

[28] Wei W., Xiang X., Peng Y., Nonlinear impulsive integro-differential equations of mixed type and optimal controls, Optimization, 2006, 55, 141-156

[29] Granas A., Dugundji J., Fixed Point Theory, Springer-Verlag, New York, 2003 